\title{
Effectiveness of Intraoperative Dexmedetomidine on Early Postoperative Cognitive Dysfunction in Elderly Patients Undergoing Video-Assisted Thoracoscopic Lobectomy: A Randomized Controlled Trial
}

\section{$\mathrm{Na} \mathrm{He}$}

The affiliated hospital of Inner Mongolia Medical University

Haixia Shi ( $\nabla$ nmgmz0312@yeah.net )

The affiliated hospital of Inner Mongolia Medical University

\section{Yajuan $\mathrm{Hu}$}

The affiliated hospital of Inner Mongolia Medical University

Alberto Uribe

The Ohio State University Wexner Medical Center

\section{Xinhua Mu}

The affiliated hospital of Inner Mongolia Medical University

\section{Fei Song}

The affiliated hospital of Inner Mongolia Medical University Jianshe Yu

The affiliated hospital of Inner Mongolia Medical University

\section{Research Article}

Keywords: dexmedetomidine, postoperative cognitive dysfunction, elderly, thoracoscopic lobectomy, inflammation, patient controlled epidural analgesia

Posted Date: December 31st, 2020

DOI: https://doi.org/10.21203/rs.3.rs-127973/v1

License: (c) (1) This work is licensed under a Creative Commons Attribution 4.0 International License. Read Full License 


\section{Abstract}

Background: Postoperative cognitive dysfunction (POCD) is the decline in cognitive ability that occurs between one week and one year following surgery. POCD has been reported to occur in $5 \%$ to $26 \%$ of elderly surgical patients and its incidence varies with demographic, clinical, and surgical variables. Previous studies have shown that dexmedetomindine (DEX), a highly selective $\mathrm{a}_{2}$ adrenergic receptor agonist, has many effects that include opioid-sparing properties, decreased required anesthetic medication, and neuroprotection in animal models. Ultimately, DEX may benefit cognitive ability in the postoperative period. Therefore, we hypothesized that intraoperative infusion of DEX would reduce early POCD and interleukin-6 (IL-6) levels in elderly surgical patients compared to placebo.

Methods: This randomized, double-blind, controlled trial enrolled 96 elderly patients that underwent videoassisted thoracoscopic lobectomy (VATL) between May 2017 and April 2018. The enrolled subjects were randomly assigned in a 1:1 ratio to receive intravenous dexmedetomidine (DEX Group) or placebo (0.9\% normal saline, Saline Group) as a continuous infusion at a rate of $0.5 \mu \mathrm{g} / \mathrm{kg} / \mathrm{hr}$ starting at anesthesia induction until incision closure. All subjects received total intravenous anesthesia (TIVA) during surgery and patient controlled epidural analgesia (PCEA) following surgery. The primary outcomes were to determine the incidence of POCD and IL-6 expression at postoperative day (POD) 1, 2, and 7. The secondary outcomes were to evaluate the degree of pain on POD 1 and 2, as well as adverse events throughout the study.

Results: 96 eligible subjects (48 per group) were randomized, dosed with study medication, and evaluated per protocol. There was a significant difference on POCD between the DEX and saline groups on POD 1 (6.3\% vs. $20.8 \%$, p-value $<0.05)$ and POD $2(4.2 \%$ vs. $16.7 \%$, p-value $<0.05)$. The IL-6 levels were significantly lower in the DEX group when compared with saline group during POD 1 and 2 (p-value $<0.05)$. IL-6 returned to baseline levels on POD 7. There was no significant difference in POCD on POD7, and overall there were no significant differences in the incidence of adverse events between both groups.

Conclusions: The administration of intraoperative DEX in elderly patients undergoing VATL decreased the incidence of early POCD.

Clinical Trial Registry: This study was registered on Chinese Clinical Trial Registry with the ID ChiCTR-IPR17010958.

\section{Introduction}

Postoperative cognitive dysfunction (POCD) is the decline in cognitive ability (especially executive functions and memory) that occurs between one week and one year after surgery [1, 2]. Early POCD has been associated with higher mortality incidences, dementia and premature retirement from the workforce [2]. POCD is a main contributing factor associated with prolonged hospitalization, quality of life (QOL) deterioration, and increased morbidity/mortality [1-4]. POCD has been reported to occur in $5-26 \%$ of elderly surgical patients and its incidence varies with demographic, clinical and surgical variables $[1,5]$. 
POCD has also been demonstrated in patients undergoing video-assisted thoracoscopic lobectomy (VATL) with one lung ventilation (OLV) [6, 7]. OLV increases the release of various cytokines due to perioperative organ injury [8].An inflammatory reaction, particularly in the central nervous system, is strongly associated with POCD $[5,8]$. A meta-analysis indicated that interleukin-6 (IL-6) could serve as a biomarker to guide the diagnosis, prevention, and treatment of POCD [9].

Dexmedetomidine (DEX), a highly selective $a_{2}$ adrenergic receptor agonist, can induce physiological sleep, inhibit inflammatory and stress response, and have brain protective effects $[1,10]$. Previous studies have shown that DEX has many effects that include opioid-sparing properties, decreased required anesthetic medication, and neuroprotection in animal models $[1,10]$. A study conducted by Monk et al. suggested that DEX prevented POCD due to its effective inhibition of the inflammatory response and neuroprotective effects both in vitro and in vivo [11]. Ultimately, DEX may benefit cognitive ability in the postoperative period [1].

We hypothesize that the intraoperative infusion of DEX will reduce POCD and IL-6 levels compared to placebo. We designed a randomized controlled trial (RCT) to assess the effect of intraoperative DEX administration on POCD and interleukin-6 (IL-6) expression in elderly patients undergoing VATL under general anesthesia and patient controlled epidural analgesia (PCEA). The primary outcomes were to assess the effect of the administration of DEX compared to placebo on POCD and expression of IL-6 in elderly patients undergoing VATL.

\section{Materials And Methods}

\section{Study design}

A prospective, randomized, double-blind, placebo controlled trial was designed and conducted at the Affiliated Hospital of Inner Mongolia Medical University, Hohhot, Inner Mongolia, China. This RCT met the guidelines of Good Clinical Practice and Declaration of Helsinki [12]. The study protocol was registered prior to patient enrollment at the Chinese Clinical Trial Registry at http://www.chictr.org.cn/abouten.aspx (Registration number: ChiCTR-IPR-17010958, Principal investigator, $\mathrm{Na} \mathrm{He}$ and Haixia Shi, Date of registration: 23/3/ 2017). This study was ethically approved by the Institution Review Board of the Affiliated Hospital of Inner Mongolia Medical University (2017-003; Hohhot, China). All patients signed written informed consent prior to surgery and study activities. This study has been described following the consolidated standards of reporting trials (CONSORT) guidelines for the presentation of clinical trials [13].

\section{Participants}

We screened patients older than 65 years old undergoing elective VATL for lung cancer between May 2017 and April 2018. All patients received total intravenous anesthesia (TIVA) and PCEA. Exclusion criteria included history of mental disorders, mental impairment (all patients were consulted by a psychiatrist one day before surgery), substance or alcohol addiction, smoking (14 days prior to surgery), 
sleep disorders, and severe chronic obstructive pulmonary disease that required oxygen therapy. Subjects with partial pressure of oxygen (PaO2) $<60 \mathrm{mmHg}$ in arterial blood, preoperative bradycardia (heart rate $(\mathrm{HR})<45 \mathrm{bpm})$, severe visual or hearing impairment, protocol violation, baseline Mini-Mental State Examination (MMSE) score $<24$, prolonged extubation during anesthesia emergence ( $>30$ minutes), and failure of PCEA (failure of epidural puncture or pain score $>5$ ) were also excluded.

\section{Preparation of study drug}

DEX was infused assuming the concentration of the study drug was $100 \mu \mathrm{g} / \mathrm{mL}$ ( $200 \mu \mathrm{g}$ were diluted in $50 \mathrm{~mL}$ of $0.9 \%$ normal saline by pharmacy assistant). Infusion rate $(\mathrm{mL} / \mathrm{hr})=$ weight $(\mathrm{kg}) \times$ Infusion Dose $(0.5 \mu \mathrm{g} / \mathrm{kg} / \mathrm{hr}) /$ Infusion concentration $(4 \mu \mathrm{g} / \mathrm{mL})$.

\section{Randomization and blinding}

Subjects were randomized in a $1: 1$ ratio to receive dexmedetomidine $0.5 \mu \mathrm{g} / \mathrm{kg} / \mathrm{hr}$ arm or saline placebo using a computer-generated randomization schedule. The pharmacist that prepared the study drug was the only un-blinded personnel. The respective continuous infusion of DEX or placebo were administered during surgery.

\section{Parameters for general anesthesia}

All patients enrolled in the study were scheduled to receive TIVA. Patients fasted overnight and without premedication. Midazolam or other benzodiazepines were avoided. After entering the operating room, electrocardiograph and pulse oxygen were monitored. Vital signs including MAP, HR, SpO2, and temperature were monitored in the operating room and recorded. During the Intra-operative Period, esophageal or other core temperature was monitored and recorded. Invasive blood pressure was monitored by a left radial artery catheter under local anesthesia. Thoracic epidural catheters were placed at T6-7 interspace and advanced 3-4 cm cephalically for PCEA prior to anesthesia induction.

Fentanyl, $0.2-0.4 \mu \mathrm{g} / \mathrm{kg}$, etomidate, $0.3 \mathrm{mg} / \mathrm{kg}$, and rocuronium $0.6 \mathrm{mg} / \mathrm{kg}$ were used to induce anesthesia. Neuromuscular blocking agents were used for intubation and anesthesia maintenance at the anesthesiologist's discretion. After double lumen intubation, mandatory volume ventilation was applied throughout the procedure with expiratory tide carbon dioxide $\left(\mathrm{ETCO}_{2}\right) 35-45 \mathrm{mmHg}$. Anesthesia maintenance consisted of propofol and remifentanil; and Bispectral index (BIS) was maintained between 40 and 60 during the surgery. Cis-atracurium titrated according to the need of surgery process. Propofol and remifentanil were discontinued at the start of skin closure, and the analgesia pump was connected. Patients were extubated after muscle relaxant reversed, conscious level achieved, and adequate tide volume gained. PCEA was started after wound closure and a loading dose of $5 \mathrm{ml} 0.15 \%$ ropivacaine was injected. Then, the PCEA with $0.15 \%$ ropivacaine and $0.12 \mu \mathrm{g} / \mathrm{ml}$ sufentanil was started. Analgesia pump parameters were set to a background flow of $5 \mathrm{ml} / \mathrm{hr}$, PCA of $3 \mathrm{ml}$ and a lockout time of $15 \mathrm{~min}$. If the patient's rest visual analogue scale (VAS) was above $3,3 \mathrm{ml}$ of analgesia pump drug was given by the investigator. PCEA was removed on the morning of POD 3.

\section{Intervention}


Subjects were randomly assigned to DEX group or Saline group (placebo). The study drug or placebo was infused using a controlled infusion device (standard intravenous pump system) that precisely infused study drug. Subjects assigned to the DEX group received a continuous infusion of DEX that was pumped through a peripheral vein assess at a rate of $0.5 \mu \mathrm{g} / \mathrm{kg} / \mathrm{hr}$ starting at anesthesia induction without bolus or loading dose and stopping when the chest closure started. Subjects in the saline group received the same dose rate of $0.9 \%$ normal saline.

\section{Outcomes}

The primary outcomes were to assess the effect of the administration of DEX compared with saline on POCD and expression of IL- 6 in elderly patients undergoing VATL. Both neurocognitive scales and serum level of IL-6 for each patient were assessed at baseline (prior to surgery) and consequently on the postoperative day (POD) 1, 2, 7. POCD was diagnosed by Z-score in a comprehensive test scale recommended by the International Study of POCD (ISPOCD) [14]. Patients were defined as having POCD when Z-score were above 1.96 using cross-reference [15].

The secondary outcomes were postoperative analgesia effect (scored using a VAS, where 0 represented no pain and 10 represented the strongest pain) and adverse events (including bradycardia, nausea or vomiting, skin itching, respiratory depression, and shiver) within 2 days after surgery.

\section{Statistical analysis}

Based on preliminary results not published, we calculated that $17 \%$ of difference with POCD between groups, 86 patients (43 each group) will provide $80 \%$ power and a type I error of 0.05 (two-sided test). In this trial, 48 patients in each group were recruited to allow for drop out. All data was processed by the statistical package, SPSS v. 22.0, for Windows (SPSS, Inc., Chicago, IL, USA). Quantitative variables were presented as mean \pm standard deviation. Incidences were analyzed using $\chi 2$ or Fisher's exact tests. Continuous variables were tested with Student's t-tests for normal distribution data; otherwise, MannWhitney $U$ tests were used. Repeat analysis of variance (ANOVA) was applied to compare the difference among the different times in the two groups. The significance level was set at $5 \%$.

\section{Results}

A total of 130 subjects were enrolled and assessed for potential participation in the study and underwent screening procedures. After eligibility assessments, 34 subjects were considered ineligible due to not meeting inclusion criteria (20), declined their participation (8) and canceled surgery (6). The remaining 96 subjects fulfilled the screening procedures and were randomized to be allocated in either group. After completing all enrollment activities and blinding removal, 48 subjects were allocated to the DEX group and 48 subjects were allocated to the saline group. The flow diagram of this RCT following the CONSORT 2010 guidelines is shown in Figure 1 [13]. Therefore, the data analysis of 96 subjects was conducted.

There were no significant differences in American Society of Anesthesiology (ASA) Physical Status Classification, age, sex, body mass index (BMI), education years, anesthesia length (from induction to 
anesthesia stop), OLV length and length of extubation after anesthesia stop found between the groups $(P>0.05)$ (Table 1).

Table 1. Baseline characteristics of patients in the DEX and saline groups

\begin{tabular}{|llll|}
\hline Parameter & DEX group $(\mathrm{n}=48)$ & Saline group $(\mathrm{n}=48)$ & $P$-value \\
\hline ASA-PS(I/II/III), $\mathrm{n}$ & $0 / 40 / 8$ & $0 / 39 / 9$ & 0.63 \\
\hline Sex, male/female, $\mathrm{n}$ & $38 / 10$ & $36 / 12$ & 0.63 \\
\hline Age, years & $68.7 \pm 4.6$ & $68.7 \pm 3.4$ & 0.98 \\
\hline Education, years & $6.1 \pm 2.9$ & $7.1 \pm 2.4$ & 0.06 \\
\hline BMI, kg/m ${ }^{2}$ & $22.2 \pm 1.5$ & $22.5 \pm 1.4$ & 0.32 \\
\hline Surgery time, min & $179.5 \pm 48.0$ & $185.9 \pm 45.2$ & 0.45 \\
\hline Anesthesia time, min & $202.2 \pm 48.4$ & $210.6 \pm 45.7$ & 0.33 \\
\hline OLV time, min & $153.5 \pm 46.1$ & $160.7 \pm 44.1$ & 0.38 \\
\hline Time to extubation, $\min$ & $9.2 \pm 2.2$ & $9.7 \pm 1.8$ & 0.09 \\
\hline
\end{tabular}

Note: Data are shown as mean \pm standard deviation (SD) or $\mathrm{n}$.

Abbreviations: ASA-PS, American Society of Anesthesiology Physical Status; OLV, one lung ventilation; DEX, Dexmedetomidine.

There was a significant difference on POCD between the DEX group and saline group on POD 1 (6.3\% vs. $20.8 \%$, p-value 0.037 ) and POD 2 (4.2\% vs. 16.7\%, p-value 0.045). (Table 2).

Table 2. Comparison of the incidence of POCD in the DEX and saline groups

\begin{tabular}{|llll|}
\hline & DEX group $(\mathrm{n}=48)$ & Saline group $(\mathrm{n}=48)$ & $P$-value \\
\hline POD 1, n (\%) & $3(6.3 \%)$ & $10(20.8 \%)$ & 0.037 \\
\hline POD 2, n (\%) & $2(4.2 \%)$ & $8 \llbracket 16.7 \%)$ & 0.045 \\
\hline POD 7, n (\%) & $0(0)$ & $0(0)$ & \\
\hline
\end{tabular}

Note: Data are expressed as $n(\%)$.

Abbreviations: POD, Post-operative day; DEX, Dexmedetomidine.

There were no significant differences on baseline IL- 6 levels between both groups. The IL-6 levels were significantly lower on the DEX group when compared with saline group during POD 1 and 2 (p-value $<0.05)$. IL-6 returned to baseline levels on POD 7. (Figure. 2) 
There were no significant differences on pain VAS scores at rest between the groups during POD 1 and 2 , while the pain VAS scores at movement were significantly lower in the DEX group ( $p$-value $<0.05$, Table 3 ).

Table 3. The degree of pain VAS scores on postoperative day 1 and 2

\begin{tabular}{|lllll|}
\hline Variables & Time points & $\begin{array}{l}\text { DEX group } \\
(\mathrm{n}=48)\end{array}$ & $\begin{array}{l}\text { Saline group } \\
(\mathrm{n}=48)\end{array}$ & $\mathrm{p}$-value \\
\hline Rest VAS & POD 1 & $0.02 \pm 0.14$ & $0.11 \pm 0.32$ & 0.051 \\
\hline Movement VAS & POD 1 & $0.96 \pm 0.19$ & $2.08 \pm 0.27$ & 0.013 \\
\hline & POD 2 & $1.94 \pm 0.23$ & $3.04 \pm 0.19$ & 0.025 \\
\hline
\end{tabular}

Note. The degree of pain is evaluated by VAS. VAS are expressed as mean \pm standard deviation (SD).

Abbreviations: VAS, the visual analogue scale; POD, Post-operative day; DEX, Dexmedetomidine.

Adverse events collected for the study (bradycardia, nausea or vomiting, skin itching, respiratory depression and shiver) were rare and there were no significant differences between the groups ( $\mathbf{p}$-value > 0.05 , Table 4).

Table 4. Adverse events within 2 days after surgery

\begin{tabular}{|llll|}
\hline & DEX group $(\mathrm{n}=48)$ & Saline group $(\mathrm{n}=48)$ & $p$-value \\
\hline bradycardia & $5(12.5 \%)$ & $3(6.7 \%)$ & 0.357 \\
\hline Nausea or vomiting & $4(8.4 \%)$ & $5(12.5 \%)$ & 0.726 \\
\hline Respiratory depression & 0 & 0 & - \\
\hline itching & & & 1.000 \\
\hline shiver & $2(4.2 \%)$ & $2(4.2 \%)$ & 1.000 \\
\hline
\end{tabular}

Note. Data are expressed as $n(\%)$.

Abbreviations: DEX, Dexmedetomidine.

\section{Discussion}


Surgical interventions can activate a cascade of reactions which could then result in an inflammatory response and pain [16]. The increase of proinflammatory cytokines may be an essential risk factor in cognitive decline after surgery [17]. Over-expression of inflammatory cytokines may lead to POCD [18].

POCD is a risk factor that results in a longer hospital stay, a lower likelihood of a return to independent living, and increased mortality [19]. An international trial of elderly patients (median age 68, range 60-81 years) demonstrated the incidence of POCD could be up to $26 \%$ within 7 days after non-cardiac surgery, and advanced age and length of anesthesia time were highly related with POCD [20]. Patients with OLV during thoracic surgery were at higher risk of developing POCD [6]. Our study selected subjects who presented with high risks, including elderly patients undergoing VATL with OLV.

DEX, an alpha 2 adrenal receptor agonist, has a range of effects on inflammation, anesthesia, analgesia, and cerebral protection. DEX used in monitored anesthesia can significantly reduce requirements for both midazolam and fentanyl, and provide better patient satisfaction [21]. DEX exhibited significant effects against oxygen and glucose deprivation-induced injury [22]. The increase of pro-inflammatory cytokines in the hippocampus may be an important risk factor for cognitive decline after surgery [23]. The aim of this study was to investigate the effectiveness of DEX on POCD and the levels of main inflammatory marker (IL-6) in elderly patients undergoing VATL for lung cancer.

This study demonstrated that the incidence of POCD on POD1 was higher than that at the other time points, with the incidence of POCD decreasing over time. This trial suggests that patients with POCD at POD1 are prone to develop POCD. Moreover, the patients were administered DEX from incision to chest closure, with no infusion occurring during the postoperative period. It is possible that the dose of intraoperative DEX was associated with the setting of the neurochemical milieu of a general anesthetic. The expression of IL-6 reached the climax on POD 1, which is consistent with the occurrence of POCD. This result might be due to the relation between the infusion time and the acting nature of the drug, or the inflammation activated by trauma.

We excluded patients with perioperative severe cardiovascular and pulmonary comorbidities, such as coronary artery disease, arrhythmia, heart dysfunction, chronic obstructive pulmonary disease, asthma, respiratory failure, which provided a homogeneous group for which we could make conclusive statements. Conversely, Deiner et al. conducted a RCT infusing dexmedetomidine $(0.5 \mu \mathrm{g} / \mathrm{kg} / \mathrm{hr}) \mathrm{vs}$. placebo during surgery and 2 hours after surgery in patient undergoing major elective cardiac surgery; then the study assessed postoperative delirium for up to 5 days and POCD at 3 and 6 months [1]. The study concluded that the infusion of dexmedetomidine at a rate of $0.5 \mathrm{mcg} / \mathrm{kg} / \mathrm{hr}$ did not prevent POCD or postoperative delirium after major cardiac surgery [1]. The dose of dexemedetomidine used for this study was similar with the dose from our study; however, our study assessed early POCD and controlled postoperative pain with PCEA.

Thoracic epidural analgesia is a gold standard for pain control after thoracic surgery. PCEA improves outcomes, including: the influence on POCD, the opioid dose and its related side effects, such as nausea 
and vomiting, skin itching, and respiratory depression [24]. Although PCEA controlled analgesia effect for both groups in our study, DEX could significantly improve pain at movement.

This result might be implicated by another trial [21]. DEX was a safe and effective primary sedative alternative [21]. Furthermore, we combined neurocognitive score and IL-6 simultaneously. We used the recommended tests by ISPOCD in the present study and calculated Z-score above 1.96 to diagnose POCD [15]. This method avoided floor or ceiling effects. The evaluators were well trained and blinded to the grouping. There were no significances between the groups in adverse events. We did not use a loading dose of DEX as the other study, but bradycardia rarely occurred.

Our study had a few limitations to be considered. First, we followed up the patients within 7 days after surgery when they were at a high risk for developing POCD. This might overlook the patients that could develop POCD after 7 days postoperatively. Second, although there were many biochemical indicators to predict POCD, we chose a popular clinical biomarker IL- 6 because of limited funding. Third, we excluded the patients with severe pulmonary disease, which may decrease the incidence of POCD. Fourth, the values of oxygen pressure in the artery blood were normal, therefore we did not collect and analyze the values in artery blood gas. Lastly, this study did not assess for postoperative delirium and that might interfere with POCD assessments.

\section{Conclusion}

The administration of intraoperative DEX at $0.5 \mu \mathrm{g} / \mathrm{kg} / \mathrm{hr}$ infusion in elderly patients undergoing VATL decreased the incidence of early POCD. In addition, PCEA provided satisfied analgesic effect. The use of DEX and PCEA might reduce the IL- 6 expression and pain VAS at movement within the first two days after surgery.

\section{Abbreviations}

Postoperative cognitive dysfunction (POCD), dexmedetomindine (DEX), video-assisted thoracoscopic lobectomy (VATL), postoperative day (POD)

\section{Declarations}

Ethics approval and consent to participate: The ethics committee of the Affiliated Hospital of Inner Mongolia Medical University approved the study protocol (no: 2017-003). All patients signed written informed consent prior to surgery and study activities.

Consent for publication: The authors consent for publication.

Availability of data and materials: The datasets used and analysed during the current study are available from the corresponding author on reasonable request. 
Competing interests: The authors declare that they have no competing interests.

Funding: This study was supported by Inner Mongolia Regional National Natural Science Fund [grant no.2017MS08107] and Inner Mongolia Funds of Medical Health committee [grant no. 201703096].

\section{Authors' contributions:}

$\mathrm{Na}$ He: This author helped perform the study and write the initial manuscript.

Haixia Shi: This author helped design the protocol and write the final manuscript.

Yajuan Hu: This author helped review the citations and analyze the data.

Alberto Uribe: This author helped revise the manuscript and edit language.

Xinhua Mu: This author helped complete the neurocognitive scale and edit the final manuscript.

Fei Song: This author helped test the expression of IL- 6 and edit the final manuscript.

Jianshe Yu: This author helped guide the study and edit the manuscript.

Acknowledgement: Thanks to Alberto Uribe and Caitlin Hatten for editing the manuscript.

\section{References}

1. Deiner S, Luo X, Lin H-M, Sessler DI, Saager L, Sieber FE, et al. Intraoperative infusion of dexmedetomidine for prevention of postoperative delirium and cognitive dysfunction in elderly patients undergoing major elective noncardiac surgery: a randomized clinical trial. JAMA Surg. 2017;152:e171505--e171505.

2. Besch G, Vettoretti L, Claveau M, Boichut N, Mahr N, Bouhake Y, et al. Early post-operative cognitive dysfunction after closed-loop versus manual target controlled-infusion of propofol and remifentanil in patients undergoing elective major non-cardiac surgery: Protocol of the randomized controlled single-blind POCD-ELA trial. Medicine (Baltimore). 2018;97.

3. Moller JT, Rasmussen LS, Hanning C. Postoperative cognitive dysfunction in the elderly. Lancet. 1998;351:1889.

4. Steinmetz J, Christensen KB, Lund T, Lohse N, Rasmussen LS, Group I. Long-term consequences of postoperative cognitive dysfunction. J Am Soc Anesthesiol. 2009;110:548-55.

5. Monk TG, Weldon BC, Garvan CW, Dede DE, Van Der Aa MT, Heilman KM, et al. Predictors of cognitive dysfunction after major noncardiac surgery. Anesthesiol J Am Soc Anesthesiol. 2008;108:18-30.

6. Li X, Li F, Liu Z, Shao M. Investigation of one-lung ventilation postoperative cognitive dysfunction and regional cerebral oxygen saturation relations. J Zhejiang Univ B. 2015;16:1042-8.

7. Nolan JP, Kelly FE. Airway challenges in critical care. Anaesthesia. 2011;66:81-92. 
8. Xie G, Zhang W, Chang Y, Chu Q. Relationship between perioperative inflammatory response and postoperative cognitive dysfunction in the elderly. Med Hypotheses. 2009;73:402-3.

9. Peng $L, X u L$, Ouyang $W$. Role of peripheral inflammatory markers in postoperative cognitive dysfunction (POCD): a meta-analysis. PLoS One. 2013;8:e79624.

10. Weerink MAS, Struys MMRF, Hannivoort LN, Barends CRM, Absalom AR, Colin P. Clinical pharmacokinetics and pharmacodynamics of dexmedetomidine. Clin Pharmacokinet. 2017;56:893913.

11. Monk TG, Price CC. Postoperative cognitive disorders. Curr Opin Crit Care. 2011;17.

12. Lewis JA, Jonsson B, Kreutz G, Sampaio C, van Zwieten-Boot B. Placebo-controlled trials and the Declaration of Helsinki. Lancet. 2002;359:1337-40.

13. Begg C, Cho M, Eastwood S, Horton R, Moher D, Olkin I, et al. Improving the quality of reporting of randomized controlled trials: the CONSORT statement. Jama. 1996;276:637-9.

14. Rasmussen LS, Larsen K, Houx P, Skovgaard LT, Hanning CD, Moller JT, et al. The assessment of postoperative cognitive function. Acta Anaesthesiol Scand. 2001;45:275-89.

15. Zhou M, Lyu Y, Zhu Y, Jiang T, Wu C, Yang J, et al. Effect of Ulinastatin Combined with Dexmedetomidine on Postoperative Cognitive Dysfunction in Patients Underwent Cardiac Surgery. Front Neurol. 2019;10:1293.

16. Cunliffe RN. a-Defensins in the gastrointestinal tract. Mol Immunol. 2003;40:463-7.

17. Cao X-Z, Ma H, Wang J-K, Liu F, Wu B-Y, Tian A-Y, et al. Postoperative cognitive deficits and neuroinflammation in the hippocampus triggered by surgical trauma are exacerbated in aged rats. Prog Neuro-Psychopharmacology Biol Psychiatry. 2010;34:1426-32.

18. Barrientos RV, Sotelo JP, Pacheco MP, Read JSB, Callejas RL. Analysis and electrical modeling of a cylindrical DBD configuration at different operating frequencies. Plasma Sources Sci Technol. 2006;15:237-45.

19. Czyz-Szypenbejl K, Medrzycka-Dabrowska W, Kwiecien-Jagus K, Lewandowska K. The occurrence of postoperative cognitive dysfunction (POCD)-systematic review. Psychiatr Pol. 2019;53:145-60.

20. Newman MF, Kirchner JL, Phillips-Bute B, Gaver V, Grocott H, Jones RH, et al. Neurological Outcome Research, G., \& the Cardiothoracic Anesthesiology Research Endeavors, I.(2001). Longitudinal assessment of neurocognitive function after coronary-artery bypass surgery. $\mathrm{N}$ Engl $\mathrm{J}$ Med. 344:395-402.

21. Candiotti KA, Bergese SD, Bokesch PM, Feldman MA, Wisemandle W, Bekker AY, et al. Monitored anesthesia care with dexmedetomidine: a prospective, randomized, double-blind, multicenter trial. Anesth Analg. 2010;110:47-56.

22. Dahmani S, Rouelle D, Gressens P, Mantz J. Characterization of the postconditioning effect of dexmedetomidine in mouse organotypic hippocampal slice cultures exposed to oxygen and glucose deprivation. Anesthesiol J Am Soc Anesthesiol. 2010;112:373-83. 
23. Hovens IB, Schoemaker RG, van der Zee EA, Absalom AR, Heineman E, van Leeuwen BL.

Postoperative cognitive dysfunction: involvement of neuroinflammation and neuronal functioning.

Brain Behav Immun. 2014;38:202-10.

24. Raft J, Richebé P. Anesthesia for thoracic ambulatory surgery. Curr Opin Anesthesiol. 2019;32:73542.

\section{Figures}

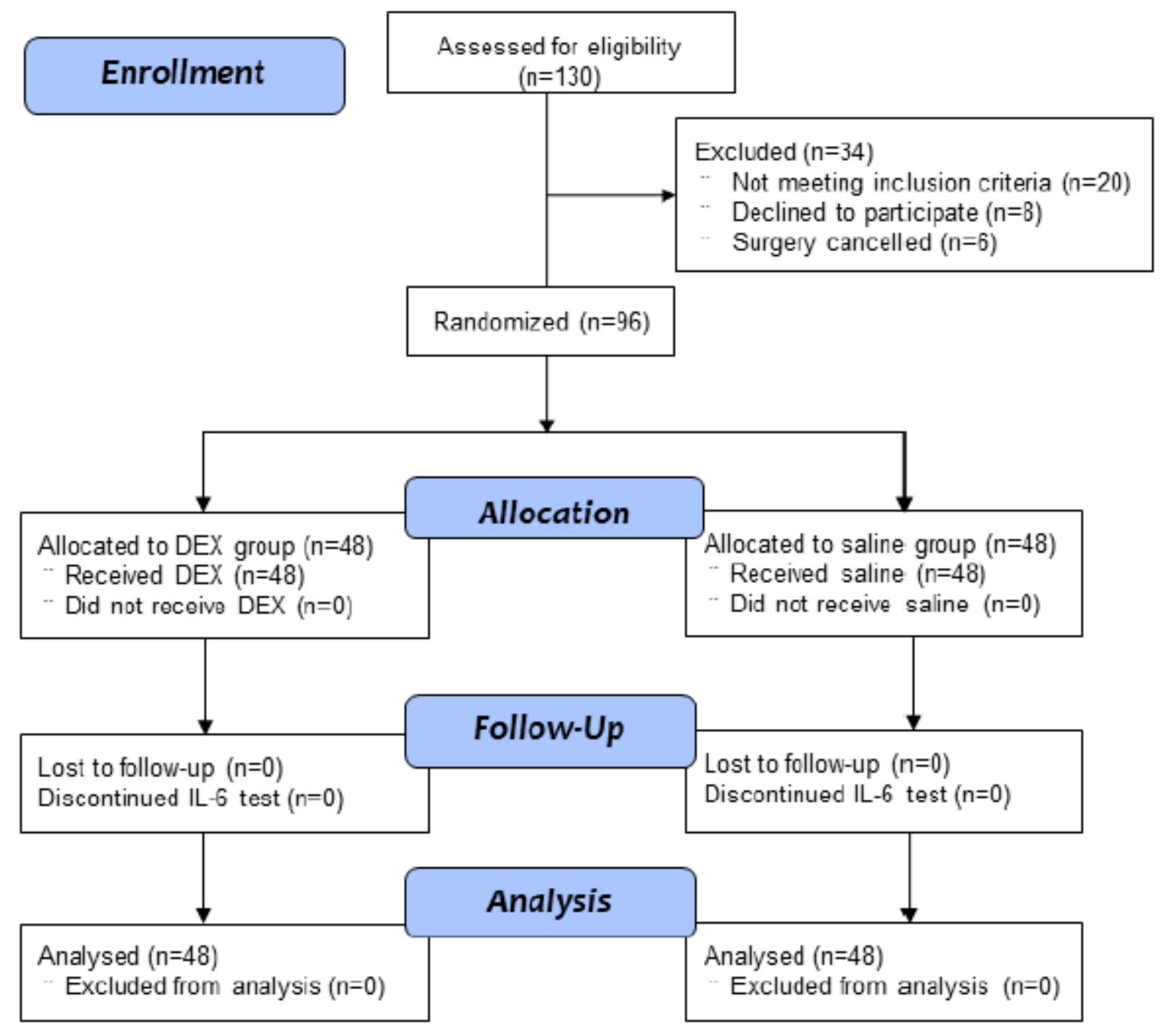

\section{Figure 1}

Trial profile according to CONSORT guidelines Abbreviations: DEX, Dexmedetomidine; IL-6, Interleukin-6. 


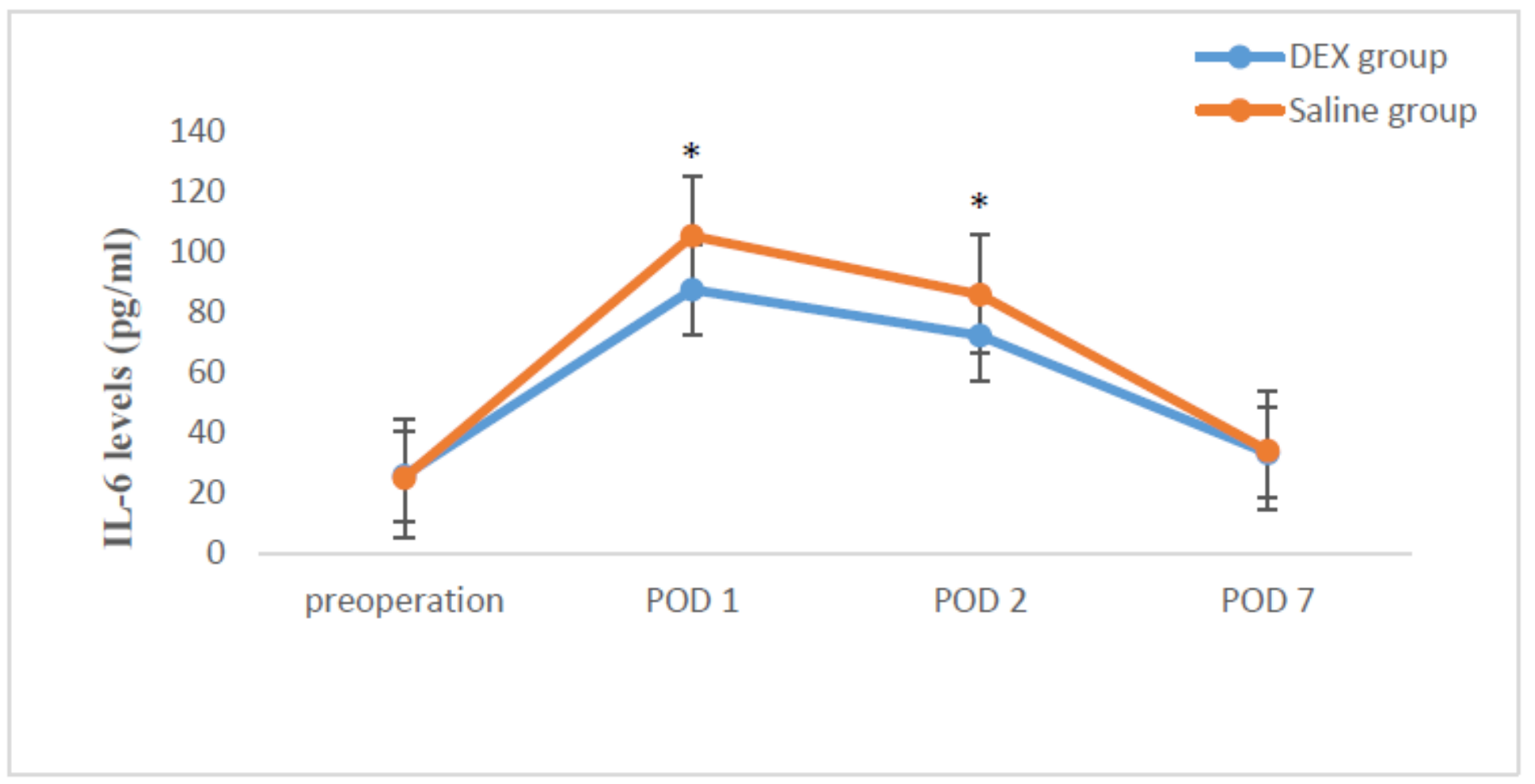

Figure 2

Comparison of IL-6 levels. Note: Data are expressed as mean \pm standard deviation (SD) and frequency. Abbreviations: POD, Post-operative day; DEX, Dexmedetomidine; IL-6, Interleukin-6. Compared with saline group, * $\mathrm{P}<0.05$. 